\title{
Reconocimiento de expresiones faciales de emociones en la enfermedad de Parkinson: una revisión teórica
}

\author{
Laura Alonso-Recio, Juan M. Serrano-Rodríguez, Fernando Carvajal-Molina, Ángela Loeches-Alonso, \\ Pilar Martín-Plasencia
}

Introducción. La expresión facial emocional constituye una guía básica en la interacción social y, por ello, las alteraciones en su expresión o reconocimiento suponen una importante limitación para la comunicación.

Objetivo. Examinar las capacidades de reconocimiento de expresiones faciales y su posible deterioro en la enfermedad de Parkinson.

Desarrollo. En primer lugar, se revisan las investigaciones sobre este tema y que no han encontrado resultados totalmente afines. En segundo lugar, se analizan los factores que pueden explicar estas divergencias y, en particular, como tercer objetivo, se valora la relación entre los problemas en el reconocimiento emocional y el deterioro cognitivo asociado a la enfermedad. Por último, se proponen estrategias alternativas para el desarrollo de estudios que contribuyan a clarificar el estado de estas habilidades en la enfermedad de Parkinson.

Conclusiones. La mayoría de los estudios indica deficiencias en el reconocimiento de expresiones, sobre todo en las de contenido emocional negativo. No obstante, es posible que dichas alteraciones estén relacionadas con las que también aparecen en otros procesos perceptivos y ejecutivos propios de la enfermedad. Para avanzar en esta cuestión, consideramos necesario diseñar estudios sobre reconocimiento emocional que impliquen diferencialmente a los procesos cognitivos mencionados o que contrasten la ejecución de los individuos con estímulos no emocionales y con expresiones faciales. Además de incrementar nuestro conocimiento sobre las consecuencias funcionales del deterioro cerebral característico de la enfermedad, estos estudios pueden indicarnos si debe prestarse atención adicional a su rehabilitación dentro de los programas que se aplican.

Palabras clave. Capacidades visuoespaciales. Deterioro cognitivo. Enfermedad de Parkinson. Expresión facial. Función ejecutiva. Reconocimiento de emociones.

\section{Introducción}

La enfermedad de Parkinson (EP), además de por los síntomas motores predominantes, se caracteriza por un conjunto de alteraciones cognitivas y emocionales. Estas alteraciones se manifiestan desde etapas tempranas de la enfermedad y en ausencia de cuadros de demencia, que aparecen con frecuencia en fases más avanzadas. Entre las alteraciones cognitivas mejor descritas se encuentran las que afectan a la función ejecutiva, a la percepción visuoespacial y a la memoria [1-5]. En cuanto a las alteraciones emocionales, junto con las que afectan al estado de ánimo general (depresión y ansiedad), se han descrito problemas en la expresión y en el reconocimiento de emociones. Respecto a este último aspecto, aunque algunos estudios han encontrado que los pacientes con EP tienen ciertos problemas en la identificación de tonos de voz con distinta prosodia emocional, la mayor parte de los estudios realizados se han centrado en las alteracio- nes en el reconocimiento de emociones a través de la expresión facial [6].

En concreto, sobre este último aspecto, las investigaciones no son unánimes sobre el alcance del deterioro en la enfermedad. Además, cabe la posibilidad de que las posibles alteraciones en el reconocimiento emocional estén vinculadas a las de los procesos cognitivos, que están asimismo implicados en el reconocimiento. El reconocimiento de emociones a través de la expresión facial, además de la identificación del contenido afectivo, implica la puesta en funcionamiento de procesos perceptivos, ejecutivos y mnésicos que, como se ha señalado con frecuencia, se han encontrado alterados en los individuos con EP.

En nuestra opinión, tratar de clarificar los déficits en el reconocimiento emocional, su alcance y su relación con el funcionamiento cognitivo presenta un doble interés. Por una parte, desde un punto de vista teórico, puede contribuir a una mejor comprensión de las repercusiones funcionales de las al-
Departamento de Psicología Biológica y de la Salud. Facultad de Psicología. Universidad Autónoma de Madrid. Madrid, España.

Correspondencia: Dra. Laura Alonso Recio. Departamento de Psicología Biológica y de la Salud. Facultad de Psicología. Universidad Autónoma de Madrid. Ciudad Universitaria de Cantoblanco. Iván Pavlov, 6. E-28049 Madrid.

E-mail:

laura.alonso@uam.es

Financiación:

Proyecto ref. PSI2009-09067, financiado por el Ministerio de Ciencia e Innovación.

Aceptado tras revisión externa: 16.01.12.

Cómo citar este artículo: Alonso-Recio L, Serrano-Rodríguez JM, Carvajal-Molina F, LoechesAlonso A, Martín-Plasencia P. Reconocimiento de expresiones faciales de emociones en la enfermedad de Parkinson: una revisión teórica. Rev Neurol 2012; 54: 479-89.

(c) 2012 Revista de Neurología 
teraciones en los circuitos cerebrales dopaminérgicos y extradopaminérgicos, que se han descrito en la EP [7]. Dichas alteraciones, que afectan principalmente a las conexiones frontoestriadas, se han relacionado con los problemas ejecutivos y visuoespaciales que muestran estos pacientes [8]. La vinculación entre las posibles alteraciones cognitivas y emocionales puede aportar datos a favor del papel de dichos circuitos frontoestriados en el procesamiento emocional. Por su parte, si el deterioro en el reconocimiento emocional ocurre con independencia del de otros procesos cognitivos, puede indicar la participación de otros circuitos cerebrales. En particular, en la EP se han descrito también cambios degenerativos en circuitos mesolímbicos y que afectan a otros neurotransmisores, como la serotonina, la noradrenalina y la acetilcolina $[9,10]$. En este mismo sentido, las investigaciones sobre las bases cerebrales de la emoción apuntan, por ejemplo, la importancia de núcleos como la amígdala en la percepción del miedo y de áreas corticales como la ínsula en el caso de la ira y el desagrado [11].

Por otra parte, la determinación de las dificultades reales para reconocer emociones en el rostro puede resultar relevante también desde un punto de vista aplicado, dada la importancia que estos elementos poseen en la interacción social. Las limitaciones en esta capacidad pueden desempeñar un papel importante en las posibilidades de comunicación de los individuos con EP con otras personas, contribuir a su aislamiento y, en definitiva, a la pérdida de calidad de vida durante la enfermedad. De este modo, su correcta valoración y diferenciación de otros posibles deterioros cognitivos puede permitir, en su caso, al diseño de programas de intervención más específicos, que contribuyan a mejorar su desenvolvimiento cotidiano y sus posibilidades de relación.

Con las consideraciones anteriores, este trabajo persigue los siguientes objetivos:

- Resumir el estado actual del conocimiento sobre las alteraciones en el reconocimiento de expresiones faciales en la EP mediante una revisión bibliográfica exhaustiva.

- Analizar algunas de las particularidades de dichos estudios que pueden explicar sus distintos resultados y que se relacionan, sobre todo, con la heterogeneidad en las características de los individuos estudiados.

- Profundizar en la relación entre el deterioro en esta capacidad y el declive que también se ha observado en ciertos procesos cognitivos (en particular, visuoespaciales y ejecutivos).

- Sugerir alguna estrategia alternativa para el de- sarrollo de estudios que contribuyan a clarificar y distinguir el estado de las capacidades de reconocimiento afectivo y de los procesos cognitivos en la EP.

\section{Desarrollo}

Para el desarrollo de nuestros objetivos, llevamos a cabo una búsqueda detallada de la literatura científica a partir de diversas bases de datos (PubMed Services, PsycInfo y Medline) hasta agosto de 2011. La búsqueda se realizó sin límite temporal (año de publicación) con los siguientes términos o palabras clave: 'enfermedad de Parkinson', 'expresión facial' y 'reconocimiento emocional'. Posteriormente, se completó mediante una búsqueda manual a partir de las referencias encontradas. Tras analizar los resúmenes, se localizaron 28 investigaciones originales publicadas entre los años 1984 y 2011, cuyo objetivo principal había sido medir las capacidades de reconocimiento de expresiones faciales emocionales en pacientes con EP, a través de diferentes tareas conductuales. De este conjunto, siete evaluaron exclusivamente la capacidad de reconocimiento de expresiones faciales, 16 incluyeron adicionalmente otras pruebas para medir el estado psicológico o cognitivo, cuatro midieron la capacidad de reconocimiento como parte del protocolo previo a la intervención para la implantación de electrodos de estimulación profunda y uno de ellos fue un metanálisis de estudios previos.

Considerando en conjunto todas las investigaciones revisadas, se ponen de manifiesto tres tendencias divergentes sobre la capacidad de reconocer expresiones faciales de emociones en la EP. Un grupo de estudios apoya la existencia de un deterioro generalizado en el reconocimiento emocional; otro conjunto sostiene que se encuentran deficiencias, pero sólo con determinadas emociones; $y$, por último, un tercer grupo ha encontrado un rendimiento similar al de la población no afectada con la que se les comparó.

Respecto a los que indican una menor capacidad global para reconocer expresiones emocionales en la EP, el estudio pionero de Scott et al [12] fue el primero en encontrar que, en comparación con el grupo control, los pacientes con EP eran menos precisos en su descripción verbal de las emociones de alegría, ira y tristeza, mostradas en forma de dibujos esquemáticos. Curiosamente también, en una de las investigaciones más recientes, Herrera et al [13] han encontrado resultados similares utilizando fotografías en lugar de dibujos, y añadiendo las expresiones 
Tabla I. Estudios que informan de deterioro generalizado en la capacidad de reconocimiento de expresiones faciales en la EP.

\begin{tabular}{|c|c|c|c|}
\hline & Emociones & Tareas & Resultados \\
\hline $\begin{array}{l}\text { Beatty et al (1989) } \\
43 \text { con EP y } 46 \text { controles }\end{array}$ & $\begin{array}{l}\text { Alegría, tristeza, } \\
\text { ira, miedo, asco, } \\
\text { sorpresa y neutra }\end{array}$ & $\begin{array}{l}\text { Tarea de identificación emocional } \\
\text { Otras tareas: } \\
\text { - Test de reconocimiento de caras de Benton } \\
\text { - MMSE } \\
\text { - BDI }\end{array}$ & $\begin{array}{l}\text { Deterioro en el reconocimiento de } \\
\text { todas las expresiones faciales evaluadas }\end{array}$ \\
\hline $\begin{array}{l}\text { Jacobs et al (1995) } \\
12 \text { con EP y } 30 \text { controles }\end{array}$ & $\begin{array}{l}\text { Alegría, tristeza, } \\
\text { ira y miedo }\end{array}$ & $\begin{array}{l}\text { Tarea de discriminación emocional y tarea } \\
\text { de correspondencia de imágenes emocionales } \\
\text { Otras: tarea de discriminación de la identidad facial }\end{array}$ & $\begin{array}{l}\text { Deterioro en el reconocimiento de todas las expresiones } \\
\text { faciales evaluadas en tareas de discriminación emocional, } \\
\text { pero no de correspondencia de imágenes emocionales }\end{array}$ \\
\hline $\begin{array}{l}\text { Breitenstein et al (1998) } \\
14 \text { con EP ( } 7 \text { en estadio I } \\
\text { y } 7 \text { en estadio II) y } \\
2 \text { grupos controles }\end{array}$ & $\begin{array}{l}\text { Alegría, ira, tristeza, } \\
\text { miedo y neutra }\end{array}$ & $\begin{array}{l}\text { Tarea de discriminación emocional } \\
\text { y tarea de identificación } \\
\text { Otras tareas: } \\
\text { - Tarea de discriminación de la identidad facial } \\
\text { - Semejanzas, figuras incompletas y dígitos } \\
\text { de WAIS } \\
\text { - Estado de ánimo }\end{array}$ & $\begin{array}{l}\text { Deterioro en el reconocimiento de todas las expresiones faciales } \\
\text { evaluadas en pacientes en estadio II. Rendimiento normal en } \\
\text { pacientes en estadio I }\end{array}$ \\
\hline
\end{tabular}

Yip et al (2003)

64 con EP

( 56 bilaterales y 8

derechos) y 64 controles

\section{Alegría, tristeza, $\quad$ Tarea de identificación y tarea} miedo, ira, de discriminación emocional sorpresa y asco Otras tareas:

- Test de organización visual de Hooper

(organización visual)

- Juicio sobre orientación de líneas (juicio visual)

- Test de Ballon (atención visual)

Deterioro en el reconocimiento de todas las expresiones faciales evaluadas, en especial para el miedo y la tristeza, en pacientes bilaterales. Deterioro en el reconocimiento de tristeza y asco en pacientes unilaterales.

El deterioro en el reconocimiento de las expresiones faciales se mantiene, incluso, tras controlar el efecto de variables visuoespaciales

Deterioro en el reconocimiento en todas las expresiones faciales evaluadas. Rendimiento menor en todas las tareas ejecutivas. Correlación significativa entre ambas medidas

Otras tareas:

- Depresión y ansiedad

- Test de los 15 objetos de Pillon

- Función ejecutiva: fluidez verbal, Stroop,

letras y números, dígitos inversos y tapping

Herrera et al 2011

(en prensa)

40 con EP y 19 controles

Alegría, tristeza,
ira, sorpresa,
asco y miedo

Tarea de identificación emocional

Otras tareas:

- Funciones ejecutivas: go/no go y TMT-B,

fluidez verbal fonológica, semántica y acciones

- Memoria: episódica verbal (inmediata

y a largo plazo), MT verbal y visual

- Atención: TMT-A y búsqueda visual

- Habilidades visuoespaciales y visuoconstructivas:

contar puntos y cubos VOPS

- Lenguaje: denominación de acciones

y nombres famosos

BDI: escala de depresión de Beck; EP: enfermedad de Parkinson; MMSE: test minimental de Folstein; MT: memoria de trabajo; TMT: Trail Making Test; VOPS: Visual Object and Space Perception test; WAIS: escala de inteligencia de Wechsler para adultos.

de miedo, asco y sorpresa. En este caso, además, los autores utilizaron una tarea más precisa de identificación verbal, en la que los pacientes debían elegir, entre un conjunto de categorías verbales, aquella que definía mejor la emoción que expresaban los modelos. Esta tarea ha sido la más frecuentemente utili- zada en este tipo de estudios (Tablas I, II y III). Como puede observarse también en la tabla I, entre las dos investigaciones citadas otras han hallado resultados similares con alguna aportación adicional, como que el deterioro puede relacionarse con un trastorno más general en la percepción de rostros [14] y que pare- 
Tabla II. Estudios que informan de deterioro específico en la capacidad de reconocimiento de expresiones faciales en la EP.

\begin{tabular}{|c|c|c|c|}
\hline & Emociones & Tareas & Resultados \\
\hline $\begin{array}{l}\text { Kan et al ( } 2002) \\
18 \text { con EP y } 24 \text { controles }\end{array}$ & $\begin{array}{l}\text { Alegría, asco, miedo, } \\
\text { tristeza y neutra }\end{array}$ & $\begin{array}{l}\text { Tarea de identificación emocional } \\
\text { Otras tareas: } \\
\text { - Tarea de discriminación de la identidad facial } \\
\text { - MMSE } \\
\text { - Figura compleja de Rey } \\
\text { - Escala de depresión de Zung }\end{array}$ & $\begin{array}{l}\text { Deterioro en el reconocimiento de las expresiones } \\
\text { de miedo y asco. Sin correlaciones significativas entre } \\
\text { reconocimiento de expresiones faciales y el resto de tareas }\end{array}$ \\
\hline $\begin{array}{l}\text { Sprengelmeyer et al (2003) } \\
36 \text { con EP ( } 20 \text { con medicación } \\
\text { y } 16 \text { sin medicación) y } \\
40 \text { controles }\end{array}$ & $\begin{array}{l}\text { Alegría, sorpresa, } \\
\text { miedo, tristeza, } \\
\text { asco e ira }\end{array}$ & $\begin{array}{l}\text { Tarea de identificación emocional } \\
\text { Otras tareas: } \\
\text { - Reconocimiento del género } \\
\text { - Identidad facial de famosos } \\
\text { - Identidad facial de no famosos } \\
\text { - Percepción de la mirada }\end{array}$ & $\begin{array}{l}\text { Deterioro en el reconocimiento de la expresión de asco } \\
\text { en pacientes con EP medicados y no medicados en } \\
\text { comparación con controles, aunque significativamente } \\
\text { mayor en pacientes sin medicación }\end{array}$ \\
\hline $\begin{array}{l}\text { Lachenal-Chevallet } \\
\text { et al ( } 2006) \\
12 \text { con EP y } 14 \text { controles }\end{array}$ & $\begin{array}{l}\text { Alegría, miedo, } \\
\text { ira, asco y neutra }\end{array}$ & $\begin{array}{l}\text { Tarea de identificación emocional } \\
\text { Otras tareas: } \\
\text { - Tarea de reconocimiento del género } \\
\text { - Mattis } \\
\text { - Test de } 15 \text { objetos } \\
\text { - Fluidez verbal } \\
\text { - Ansiedad }\end{array}$ & $\begin{array}{l}\text { Deterioro en el reconocimiento de las expresiones de } \\
\text { miedo y asco. Deterioro de la fluidez verbal categorial. } \\
\text { Efecto significativo de la ansiedad }\end{array}$ \\
\hline $\begin{array}{l}\text { Suzuki et al ( } 2006) \\
12 \text { con EP y } 39 \text { controles }\end{array}$ & $\begin{array}{l}\text { Alegría, miedo, } \\
\text { ira, asco, tristeza } \\
\text { y sorpresa }\end{array}$ & $\begin{array}{l}\text { Tarea de identificación emocional } \\
\text { Otras tareas: } \\
\text { - Tarea de identificación facial y tasación de género } \\
\text { - Cociente intelectual } \\
\text { - MMSE } \\
\text { - Depresión de Zung }\end{array}$ & Deterioro en el reconocimiento de la expresión de asco \\
\hline $\begin{array}{l}\text { Lawrence et al ( } 2007) \\
17 \text { con EP y } 21 \text { controles }\end{array}$ & $\begin{array}{l}\text { Ira, asco, miedo, } \\
\text { tristeza, alegría } \\
\text { y sorpresa }\end{array}$ & $\begin{array}{l}\text { Tarea de identificación emocional } \\
\text { Otra tarea: test de reconocimiento facial de Benton }\end{array}$ & Deterioro en el reconocimiento de la expresión de ira \\
\hline $\begin{array}{l}\text { Ariatti et al ( } 2008) \\
27 \text { con EP y } 68 \text { controles }\end{array}$ & $\begin{array}{l}\text { Ira, asco, miedo, } \\
\text { tristeza, alegría } \\
\text { y sorpresa }\end{array}$ & $\begin{array}{l}\text { Tarea de identificación emocional, tarea de correspondencia } \\
\text { imagen-etiqueta y tarea de correspondencia de imágenes } \\
\text { emocionales } \\
\text { Otras tareas: reconocimiento de identidad facial } \\
\text { y selección de identidad facial }\end{array}$ & $\begin{array}{l}\text { Deterioro en el reconocimiento de las expresiones } \\
\text { de tristeza y miedo en todas las tareas emocionales }\end{array}$ \\
\hline
\end{tabular}

ce afectar paralelamente a la imaginación y la expresión de las mismas emociones [15]. En este último estudio, se utilizó una tarea de discriminación visual que también se ha utilizado con frecuencia en este tipo de trabajos, en la que los individuos deciden si dos rostros mostrados simultáneamente expresan o no la misma emoción.

Otro grupo de investigaciones ha encontrado deterioro en el reconocimiento de expresiones faciales, pero que afecta solo a determinadas emociones (Tabla II). La expresión emocional que se ha encontrado alterada con más frecuencia es la de desagrado o asco, bien de forma exclusiva [16,17] o bien junto con la de miedo $[18,19]$. No obstante, otros estudios informan de alteraciones en el reconocimiento de otras expresiones, como las de ira y el miedo (y no de alegría, tristeza y sorpresa [20]) o las de ira y la sorpresa (y no de alegría, desagrado, miedo y tristeza [21]). En todos estos estudios, la metodología empleada para la evaluación de las capacidades de reconocimiento fue la de identificación emocional antes comentada, y que consistía en la elección de las categorías verbales que mejor describían determinadas emociones expresadas a través del rostro.

Por último, otro conjunto de estudios no han encontrado deterioro en las capacidades para reconocer expresiones emocionales en los pacientes con EP (Tabla III). El primer trabajo en esta línea fue el de Madeley et al [22], utilizando estímulos no validados, como eran las expresiones de los propios participantes. Posteriormente, otros estudios han llegado a conclusiones similares con tareas de reconocimiento más exhaustivas y expresiones validadas. Por ejemplo, Adolphs et al [23] y Pell y Leonard [24] no encontraron problemas en el reconocimiento, tanto con tareas de discriminación como de identificación, que requerían, además, una valoración acer- 
Tabla II. Estudios que informan de deterioro específico en la capacidad de reconocimiento de expresiones faciales en la EP (cont.).

\begin{tabular}{|c|c|c|c|}
\hline & Emociones & Tareas & Resultados \\
\hline $\begin{array}{l}\text { Martins et al (2008) } \\
17 \text { con EP y } 20 \text { controles }\end{array}$ & $\begin{array}{l}\text { Alegría, miedo, } \\
\text { tristeza, ira y sorpresa }\end{array}$ & Tarea de identificación emocional & $\begin{array}{l}\text { Deterioro en el reconocimiento de las expresiones } \\
\text { de miedo e ira }\end{array}$ \\
\hline $\begin{array}{l}\text { Clark et al ( } 2008) \\
20 \text { con EP y } 23 \text { controles }\end{array}$ & $\begin{array}{l}\text { Ira, asco, miedo, } \\
\text { alegría, tristeza, } \\
\text { sorpresa y neutra }\end{array}$ & $\begin{array}{l}\text { Tarea de identificación emocional } \\
\text { Otras tareas: } \\
\text { - Tarea de identificación de paisajes } \\
\text { - Depresión }\end{array}$ & $\begin{array}{l}\text { Deterioro en el reconocimiento de las expresiones de ira y } \\
\text { sorpresa, incluso después de controlar el efecto de la depresión. } \\
\text { Rendimiento normal en la identificación de paisajes }\end{array}$ \\
\hline $\begin{array}{l}\text { Assogna et al ( } 2010) \\
70 \text { con EP y } 70 \text { controles }\end{array}$ & $\begin{array}{l}\text { Ira, alegría, tristeza, } \\
\text { miedo, asco y neutra }\end{array}$ & $\begin{array}{l}\text { Tarea de identificación emocional } \\
\text { Otras tareas: } \\
\text { - MMSE } \\
\text { - Inventario de depresión de Beck } \\
\text { - Memoria: test de Rey de aprendizaje de palabras, } \\
\text { figura compleja de Rey } \\
\text { - Función ejecutiva: fluidez verbal fonológica, } \\
\text { WCST, Stroop }\end{array}$ & $\begin{array}{l}\text { Deterioro en el reconocimiento de la expresión facial de asco. } \\
\text { Este rendimiento se relaciona con déficit en algunos dominios } \\
\text { neuropsicológicos (memoria verbal y visuoespacial, atención, } \\
\text { praxis y fluidez verbal) }\end{array}$ \\
\hline $\begin{array}{l}\text { Martínez-Corral et al (2010) } \\
31 \text { con EP (12 con apatía y } \\
19 \text { sin apatía) y } 16 \text { controles }\end{array}$ & $\begin{array}{l}\text { Alegría, tristeza, ira, } \\
\text { miedo, sorpresa y asco }\end{array}$ & Tarea de identificación emocional & $\begin{array}{l}\text { Deterioro en el reconocimiento de expresiones de miedo, } \\
\text { ira y tristeza en pacientes con apatía. Sin deterioro en el } \\
\text { reconocimiento de expresiones faciales en pacientes } \\
\text { con EP sin apatía }\end{array}$ \\
\hline $\begin{array}{l}\text { Clark et al ( } 2010) \\
16 \text { con EP y } 20 \text { controles }\end{array}$ & $\begin{array}{l}\text { Siete emociones: } \\
\text { alegría, tristeza, asco, } \\
\text { miedo, ira, sorpresa } \\
\text { y neutra }\end{array}$ & $\begin{array}{l}\text { Tarea de identificación emocional } \\
\text { Otras tareas: } \\
\text { - Tarea de identificación de paisajes } \\
\text { - Tarea de habilidades visuales básicas: } \\
\text { agudeza visual y sensibilidad de contraste } \\
\text { - Test de reconocimiento de caras de Benton } \\
\text { - Tareas de función ejecutiva: TMT y Stroop }\end{array}$ & $\begin{array}{l}\text { Deterioro en el reconocimiento de ira en pacientes izquierdos } \\
\text { y sorpresa en derechos. Sin diferencias en el resto de tareas. } \\
\text { Sin correlación entre éstas y el reconocimiento emocional }\end{array}$ \\
\hline
\end{tabular}

EP: enfermedad de Parkinson; MMSE: test minimental de Folstein; TMT: Trail Making Test; WCST: test de clasificación de tarjetas de Wisconsin.

ca de la intensidad con la que se representaba la emoción. Asimismo, se han encontrado capacidades preservadas en pacientes que iban a someterse a intervenciones para la implantación de electrodos de estimulación cerebral profunda [25-28]. Después de la intervención, los resultados de estos estudios fueron, sin embargo, dispares, ya que en algún caso se observó un mantenimiento de la capacidad para reconocer emociones, mientras que el resto hallaron un deterioro significativo, pero solamente en la identificación de las expresiones de miedo o tristeza (Tabla III).

\section{Factores que pueden intervenir en las discrepancias encontradas sobre la capacidad para el reconocimiento emocional en la EP}

Como se ha podido comprobar, los estudios encontrados arrojan resultados dispares en relación con las capacidades de reconocimiento emocional en sujetos con EP. No obstante, la mayor parte de ellos (21 de los 28) señalan alteraciones generalizadas o que afectan a la identificación o discriminación de determinadas emociones. A este respecto, a partir de un metaanálisis de buena parte de los estudios revisados aquí, Gray y Tickle-Degnen [6] detallan que la única alteración en el reconocimiento que resulta estadísticamente significativa en la EP es la que concierne al reconocimiento de expresiones faciales de emociones negativas. No obstante, antes de dar por válida esta conclusión, hay que matizar que existen notables diferencias entre los estudios, que pueden contribuir a explicar la cierta inconsistencia que se observa en sus resultados. Por ejemplo, estas diferencias se observan cuando se comparan entre ellos el estadio de deterioro de los pacientes estudiados, el origen de los síntomas motores característicos de la enfermedad (unilateral o bilateral), el tratamiento farmacológico al que estaban siendo sometidos o el estado psicológico (presencia o no de depresión). Como se detalla a continuación, las variaciones en los estudios en cuanto a estas características han dado también lugar a conclusiones distintas y ello, a su vez, puede contribuir al conocimiento sobre las circunstancias precisas en las que el reconocimiento emocional puede verse afectado en la EP.

En concreto, respecto a la influencia del estadio de deterioro en el que se encuentran los pacientes, 
Tabla III. Estudios que informan de capacidades preservadas en el reconocimiento de expresiones faciales en la EP.

\begin{tabular}{|c|c|c|c|}
\hline & Emociones & Tareas & Resultados \\
\hline $\begin{array}{l}\text { Madeley et al (1995) } \\
9 \text { con EP }\end{array}$ & & $\begin{array}{l}\text { Tarea de producción emocional } \\
\text { Tarea de identificación emocional }\end{array}$ & $\begin{array}{l}\text { Deterioro en la producción de expresiones } \\
\text { faciales. Sin deterioro en el reconocimiento } \\
\text { de expresiones faciales }\end{array}$ \\
\hline $\begin{array}{l}\text { Adolphs et al (1998) } \\
17 \text { con EP y } 13 \text { controles }\end{array}$ & $\begin{array}{l}\text { Alegría, sorpresa, } \\
\text { miedo, ira, asco } \\
\text { y tristeza }\end{array}$ & $\begin{array}{l}\text { Tarea de identificación emocional } \\
\text { Otras tareas: } \\
\text { - Tarea de reconocimiento facial de Benton } \\
\text { - Inventario de depresión de Beck } \\
\text { - NART }\end{array}$ & $\begin{array}{l}\text { Sin deterioro en el reconocimiento de } \\
\text { expresiones faciales. Sin correlación entre } \\
\text { el rendimiento en la tarea de identificación } \\
\text { emocional y las tareas de Benton, NART e } \\
\text { inventario de depresión de Beck }\end{array}$ \\
\hline $\begin{array}{l}\text { Dujardin et al ( } 2004 \mathrm{~b}) \\
12 \text { con EP quirúrgicos } \\
\text { y } 12 \text { controles }\end{array}$ & Asco, ira y tristeza & $\begin{array}{l}\text { Tarea de identificación emocional } \\
\text { Otras tareas: } \\
\text { - Depresión y ansiedad } \\
\text { - Test de los } 15 \text { objetos de Pillon } \\
\text { - Función ejecutiva: fluidez verbal, Stroop, } \\
\text { letras y números, dígitos inversos y tapping }\end{array}$ & $\begin{array}{l}\text { Antes de la intervención, sin deterioro en el } \\
\text { reconocimiento de expresiones faciales en } \\
\text { los pacientes antes de la cirugía. Además, } \\
\text { deterioro en las tareas de función ejecutiva } \\
\text { Después de la intervención, deterioro en el } \\
\text { reconocimiento de las expresiones de ira y } \\
\text { tristeza }\end{array}$ \\
\hline
\end{tabular}

$\begin{array}{lll}\text { Biseul et al (2005) } & \text { Alegría, tristeza, } & \text { Tarea de identificación emocional } \\ 15 \text { con EP quirúrgicos } & \text { miedo, sorpresa, } & \text { Otras tareas: } \\ & \text { asco, ira y neutra } & - \text { Test de reconocimiento de caras de Benton } \\ & \text { - Mattis } \\ & - \text { MMSE } \\ & \text { - Función ejecutiva: WCST, fluidez verbal } \\ & \text { fonológica y semántica, y Stroop }\end{array}$

Sin deterioro en el reconocimiento de expresiones faciales

Pell y Leonard (2005) 21 con EP y 21 controles
Alegría, sorpresa, tristeza, ira y asco
Tarea de discriminación emocional y tarea de identificación emocional Otras tareas:

- Test de discriminación de la forma

- Test de reconocimiento de caras de Benton

- Función ejecutiva: dígitos inversos, test del trazo de color, WCST, atención de la escala de Mattis

- Depresión

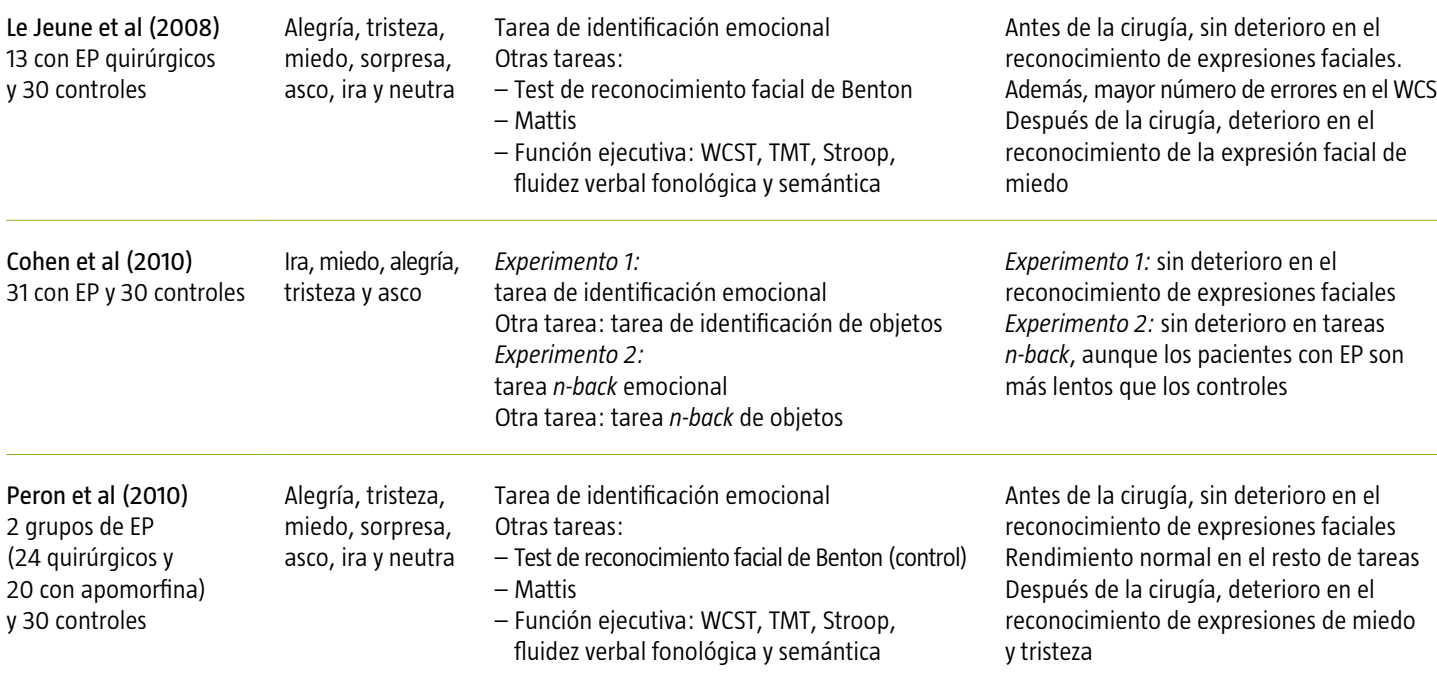

EP: enfermedad de Parkinson; MMSE: test minimental de Folstein; NART: National Adult Reading Test; TMT: Trail Making Test; WCST: test de clasificación de tarjetas de Wisconsin. 
algunos estudios informan de que las alteraciones en el reconocimiento emocional se observan desde fases iniciales de la enfermedad [29]. Sin embargo, Breitenstein et al [30], diferenciando entre individuos que se encontraban en estos estadios iniciales (estadio I frente a II, siguiendo la escala de Hoehn y Yahr [31]), sólo observaron deterioro en los que se encontraban en el estadio II. A estos resultados hay que contraponer, además, los que han encontrado capacidades preservadas en distintas etapas de la enfermedad (Tabla III). Una indefinición semejante arrojan los estudios que han controlado la influencia en el reconocimiento emocional del origen unilateral (derecho-izquierdo) o bilateral de los síntomas motores en el reconocimiento emocional. Los pocos estudios que han controlado este efecto han hallado diferentes relaciones entre el origen de la sintomatología motora y las alteraciones en el reconocimiento de expresiones faciales [32,33].

En relación con la influencia de la medicación antiparkinsoniana, los estudios que han comparado pacientes medicados y no medicados observan que la ausencia de tratamiento produce un deterioro más acusado en el reconocimiento emocional, tanto en estadios iniciales como avanzados de la enfermedad $[29,34,35]$. No obstante, otros trabajos realizados sólo con grupos de pacientes sometidos a tratamiento farmacológico también han encontrado deterioro, aunque en estos casos no se detalló o no se diferenció el efecto en relación con el estadio de desarrollo de la enfermedad $[14,18,20,21,32]$. Considerando, en conjunto, tanto el efecto de la medicación como el estadio de desarrollo de la enfermedad, la revisión de los estudios deja entrever que la manifestación de alteraciones en el reconocimiento aumenta tanto con la gravedad de los síntomas como con la ausencia de medicación, si bien esta sola constatación aporta escasa información adicional en el contexto de un trastorno con manifestaciones clínicas que son progresivamente más graves e incapacitantes.

En cuanto a la posible influencia del estado de ánimo general de los pacientes (síntomas depresivos, de ansiedad, apatía, etc.), también los resultados son algo divergentes. Algunos estudios han encontrado alteraciones en el reconocimiento emocional en pacientes que cumplían criterios de depresión o correlaciones positivas entre el estado de ánimo y el deterioro en el reconocimiento emocional [14,19, 29]. En esta línea, Martínez-Corral et al [36] encontraron también que la presencia de apatía de los pacientes con EP se relacionaba con el deterioro selectivo en el reconocimiento de rostros de miedo, ira y tristeza. Por el contrario, otros han observado un peor reconocimiento en pacientes sin sintomatología depresiva $[17,30]$ o ausencia de relación entre ambas medidas [18].

De la disparidad que se observa entre los estudios al valorar los efectos de estos factores que pueden influir en el reconocimiento emocional, se desprenden pocas conclusiones firmes. Si acaso, se apunta una casi obvia tendencia a que la gravedad de la sintomatología hace más probable la aparición de un deterioro en el reconocimiento emocional. Por ello, tal vez la conclusión principal que se deriva de ellos es la necesidad de desarrollar diseños de investigación que hagan más homogéneas las características de los grupos de EP, en cuanto a los factores mencionados y a otros que puedan introducir variabilidad en las muestras.

\section{Relación entre el deterioro cognitivo y el reconocimiento de expresiones faciales}

Aparte de la variabilidad en las características de los grupos de individuos con EP, de la revisión de los estudios sobre reconocimiento emocional se desprende otra posible fuente de explicación de las diferencias en sus resultados, que en nuestra opinión puede ser de notable relevancia. En concreto, nos referimos a la incidencia que sobre dicho reconocimiento tiene el nivel de funcionamiento de las capacidades cognitivas y que suele verse afectado en diferentes facetas en el curso de la enfermedad. Como se ha mencionado, se han empleado básicamente dos tareas para evaluar las capacidades de reconocimiento de emociones: de discriminación y de identificación (incluyendo o no una valoración de la intensidad emocional). Gray y Tickle-Degnen [6], a partir de su revisión de estudios, señalan diferencias entre ellas en cuanto a su sensibilidad para detectar deterioro en el reconocimiento de los individuos con EP. Dichas diferencias atribuibles a la tarea utilizada pueden explicarse por su distinta dificultad y, en definitiva, remiten a que cada una de ellas puede estar poniendo en funcionamiento distintos procesos y capacidades cognitivas, que interactúan con las propias del reconocimiento emocional. En otras palabras, cabe la posibilidad de que el grado de operatividad de los procesos cognitivos no estrictamente emocionales, pero que son necesarios para la identificación y para la discriminación del contenido afectivo de los rostros, desempeñe un papel relevante en la explicación de los resultados encontrados. Se trata, en suma, de precisar si el deterioro en el reconocimiento emocional es consecuencia de (o interactúa con) un declive cognitivo más global o si, por el contrario, es un fenómeno 
independiente del deterioro cognitivo y una característica distintiva a la que debe prestarse una atención principal.

Del conjunto de alteraciones cognitivas descritas en la EP destacan dos aspectos del funcionamiento cognitivo que sobresalen en cuanto a su posible implicación en el reconocimiento de expresiones faciales: por una parte, la capacidad perceptiva visuoespacial, en relación con la tarea de discriminación; y, por otra, los procesos ejecutivos (en particular, de memoria de trabajo, atención y categorización), que parecen necesarios en la resolución de la tarea de identificación emocional. Con respecto a las tareas de discriminación de expresiones, se trata de una prueba en la que el individuo se confronta con dos expresiones faciales presentadas de forma simultánea, debiendo explorar con detalle los rasgos relevantes que configuran cada una de ellas, para juzgar su similitud o diferencia. Las expresiones faciales se caracterizan por ser estímulos en los que las relaciones espaciales entre los rasgos faciales proporcionan las claves más relevantes para el reconocimiento, por encima, en muchos casos, de características aisladas del rostro [37]. De ello, se deduce que para los juicios de discriminación no basta con el contraste de detalles parciales de ambos rostros, sino llevar a cabo una exploración pormenorizada de ambas expresiones. Por ello, parece coherente pensar que una posible alteración que afecte a la manera en la que el individuo examina activamente el espacio visual influya en esta tarea de discriminación de expresiones.

En relación con la integridad de las capacidades visuoespaciales en estudios sobre discriminación emocional, hemos localizado únicamente un estudio que haya considerado la posible relación entre ambos aspectos. En concreto, Yip et al [33] administraron un conjunto de tareas de organización, juicio y atención visuoespacial, junto con las de discriminación emocional. A través de un análisis de regresión, concluyeron que el deterioro en la discriminación de expresiones faciales que encontraron no parecía afectado por el estado de las funciones visuoespaciales. En todo caso, antes de concluir que no existe esa relación entre ambos aspectos, hay que remarcar que se trata de un único estudio en el que, además, no se informó de si el grupo con EP realizó peor las pruebas visuoespaciales que el grupo control. De forma añadida, hay que señalar que los tests utilizados para evaluar estas capacidades fueron completamente diferentes de los que se utilizaron para medir el reconocimiento emocional, lo que presumiblemente limita la interpretación de sus resultados. Esto es, la distinta naturaleza de las pruebas impide conocer si son precisamente los mismos procesos perceptivos visuoespaciales los que se ponen en funcionamiento en ambas.

Respecto a la tarea de identificación emocional empleada en la mayoría de los estudios sobre reconocimiento emocional, como se indicó anteriormente, se trata de una prueba en la que el mantenimiento de determinados componentes de la función ejecutiva puede desempeñar un papel relevante. En ella, los individuos para resolver la tarea deben establecer la relación entre la imagen de una expresión facial y su significado afectivo a través del acceso a sus almacenes de memoria y, seguidamente, tomar la decisión sobre la etiqueta verbal que se corresponde con dicha expresión. Es presumible que esta secuencia requiera la puesta en funcionamiento de procesos ejecutivos como la memoria de trabajo, la atención, la capacidad de categorización y de toma de decisiones, por señalar los más evidentes [38]. Por tanto, parece muy conveniente descartar la posibilidad de que una posible alteración en alguna de estas funciones ejecutivas esté influyendo en el reconocimiento emocional, cuando se emplean tareas de identificación.

Algunos estudios han considerado esta posibilidad correlacionando las puntuaciones de los tests de función ejecutiva y las pruebas de identificación de expresiones faciales. De hecho, varios estudios han encontrado correlaciones estadísticamente significativas en individuos con EP, cuando tanto el reconocimiento de expresiones como la función ejecutiva se encontraban deteriorados en comparación con los individuos sanos [13,16,19,29]. También otro estudio ha encontrado correlación entre ambas en pacientes que mostraron deterioro en el reconocimiento emocional y un rendimiento ejecutivo comparable al del grupo control [32]. Incluso, Le Jeune et al [27] hallaron deterioro en una medida ejecutiva (número de errores del test de clasificación de cartas de Wisconsin) con capacidades de reconocimiento emocional preservadas.

Al margen de los distintos resultados encontrados, y en la línea de lo expresado anteriormente, resulta difícil interpretar el significado de la presencia o ausencia de correlaciones entre el rendimiento en pruebas estandarizadas de función ejecutiva y las específicamente diseñadas para medir la identificación emocional. Las correlaciones en estos casos proporcionan sólo un indicio de la implicación de ciertos procesos ejecutivos que es probable que sean compartidos por ambas tareas. Sin embargo, además de que es igualmente probable que difieran en otras de las capacidades necesarias para la resolución de cada una de ellas, tampoco permiten es- 
pecificar qué procesos ejecutivos son los que están explicando esa relación. En suma, la correspondencia que se encuentra entre pruebas que son netamente distintas indica sólo una medida indirecta, que no precisa y valora todos los procesos cognitivos que se ponen en funcionamiento en ambas.

Como una forma de avanzar y profundizar sobre esta limitación, dos estudios muy recientes han analizado, desde una perspectiva complementaria, la relación entre el reconocimiento emocional y el funcionamiento ejecutivo en pacientes con EP. Para ello, han optado por utilizar una sola prueba para evaluar simultáneamente el funcionamiento ejecutivo y el reconocimiento de expresiones faciales emocionales. En primer lugar, Cohen et al [39] analizaron el reconocimiento con tareas de categorización (de expresiones faciales y de objetos) y de memoria de trabajo (expresiones faciales y objetos, mediante una tarea $n$-back). Los individuos con EP fueron igual de precisos que los controles tanto al categorizar como al reconocer expresiones faciales y objetos presentados previamente, si bien fueron más lentos con ambos tipos de estímulos en las tareas de memoria de trabajo. De estos resultados, cabe resaltar únicamente que la velocidad de ejecución o de procesamiento de información debe ser considerada como un factor adicional importante en los estudios de este tipo, al juzgar el posible deterioro en las capacidades cognitivas o de reconocimiento emocional.

Por su parte, García-Rodríguez et al [40] analizaron la influencia de la atención dividida en el reconocimiento emocional mediante dos tareas, una de reconocimiento emocional y otra de tipo ejecutivo (bloques de Corsi). Sus resultados mostraron que los pacientes con EP identificaron peor las expresiones faciales que los controles, cuando se exigía prestar atención simultánea a ambas. Este estudio indica, por tanto, que el deterioro en el reconocimiento emocional se produce en unas condiciones que exigen un esfuerzo ejecutivo adicional (atencional), indicando que, al menos en estos casos, los recursos atencionales limitan la capacidad de reconocimiento emocional de los individuos con EP en relación con los del grupo control.

\section{Conclusiones}

A lo largo de esta revisión de estudios, hemos tratado de poner de manifiesto las dificultades para comparar los estudios sobre reconocimiento de expresiones faciales de emociones y extraer de ellos conclusiones firmes. Las diferencias en los diseños y en las características de los individuos con EP es- tudiados en cuanto a la gravedad y origen de sus síntomas, el estatus de medicación y el estado de ánimo pueden explicar sus diferentes conclusiones. Por ello, una recomendación básica para futuros estudios en este ámbito es la de procurar una selección de los grupos de pacientes que sea lo más homogénea posible. En todo caso, conviene señalar que esta diversidad en los resultados no es del todo sorprendente si se compara con las que también muestran los estudios que han abordado en profundidad otros aspectos del funcionamiento cognitivo en la EP. Por ejemplo, en relación directa con los presuntos procesos cognitivos implicados en el reconocimiento emocional, puede señalarse que hoy en día no existe un consenso pleno sobre qué procesos ejecutivos están específicamente alterados en la EP $[3,41]$. Es posible que la variabilidad en el grado de deterioro de ciertos aspectos del funcionamiento cognitivo sea un aspecto consustancial a la enfermedad, pero, en todo caso, indica la necesidad de investigar con mayor profundidad y precisión estas consecuencias funcionales (cognitivas y emocionales) del deterioro cerebral en la EP.

Por este motivo, y, sobre todo, para conocer el estado real de las capacidades en la EP, parece necesario conocer y poner en relación el estado de funcionamiento de los procesos cognitivos (en especial, visuoespaciales y ejecutivos) con los implicados en el reconocimiento emocional. La vinculación entre estos aspectos se ha basado en la mayor parte de los estudios en el establecimiento de correlaciones entre tareas cognitivas y emocionales claramente distintas. Alternativamente, una forma más precisa de estudiar esta relación es la utilización de diseños de investigación, en los que se empleen pruebas similares para evaluar el funcionamiento cognitivo y el reconocimiento emocional. De esta forma, pueden obtenerse medidas directas y comparables del rendimiento cognitivo con estímulos no emocionales y con expresiones faciales emocionales. Incluso, permiten variar la dificultad de las tareas y, con ello, su exigencia cognitiva, con el fin de determinar las circunstancias precisas en las que pueden manifestarse los posibles deterioros en el reconocimiento emocional. Hasta el momento, sólo dos estudios muy recientes $[39,40]$ se han enfocado en esta línea, si bien tratando aspectos muy concretos del funcionamiento ejecutivo (memoria de trabajo y atención dividida, respectivamente). Sus resultados apuntan a que las relaciones pueden ser complejas, puesto que pueden estar, a su vez, mediadas por otros aspectos, como la velocidad de ejecución o procesamiento y la dificultad de las tareas. Ello invita a continuar investigando en el papel que pueden de- 
sempeñar estos y otros procesos ejecutivos y visuoespaciales, por ejemplo, de atención selectiva, inhibición y toma de decisiones, que también pueden estar vinculados al reconocimiento emocional.

El interés de este tipo de investigaciones puede contribuir al conocimiento sobre los mecanismos cerebrales subyacentes a la enfermedad y los implicados en el reconocimiento emocional. Además, puede aportar información valiosa desde el punto de vista del abordaje de la EP en contextos clínicos, lo cual resulta de especial interés si se considera el valor comunicativo y facilitador de la interacción que posee la expresión facial emocional y, por tanto, su potencial repercusión en la actividad social cotidiana de los individuos con EP.

\section{Bibliografía}

1. Foltynie T, Brayne CEG, Robbins TW, Barker RA. The cognitive ability of an incident cohort of Parkinson's patients in the UK The CamPaIGN study. Brain 2004; 127: 550-60.

2. Janvin C, Aarsland D, Larsen JP, Hugdahl K. Neuropsychological profile of patients with Parkinson's disease without dementia. Dement Geriatr Cogn Disord 2003; 15: 126-61.

3. Kehagia AA, Barker RA, Robbins TW. Neuropsychological and clinical heterogeneity of cognitive impairment and dementia in patients with Parkinson's disease. Lancet Neurol 2010; 9: 1200-13.

4. Levin BE, Katzen HL. Early cognitive changes and nondementing behavioral abnormalities in Parkinson's disease. Adv Neuro 2005; 96: 84-94.

5. Muslimovic D, Post B, Speelman JD, Schmand B. Cognitive profile of patients with newly diagnosed Parkinson disease. Neurology 2005; 65: 1239-45.

6. Gray HM, Tickle-Degnen L. A meta-analysis of performance on emotion recognition tasks in Parkinson's disease. Neuropsychology 2010; 24: 176-91.

7. Baev KV, Greene KA, Marciano FF, Samanta JES, Shetter AG, Smith KA, et al. Physiology and pathophysiology of corticobasal ganglia-thalamocortical loops: theoretical and practical aspects. Prog Neuropsychopharmacol Biol Psychiatry 2002; 26: 771-804.

8. Pillon B, Boller F, Levy R, Dubois B. Cognitive deficits and dementia in Parkinson's disease. In Boller F, Cappa S, eds. Aging and dementia. Vol. 6. Amsterdam: Elsevier Science; 2001. p. 311-71.

9. Deblieck C, Wu AD. Neuroimaging of nonmotor features of Parkinson's disease. Rev Neurol Dis 2008; 5: 125-33.

10. Rodríguez-Oroz MC, Jahanshahi M, Krack P, Litvan I, Macías R, Bezard E, et al. Initial clinical manifestations of Parkinson's disease: features and pathophysiological mechanisms. Lancet Neurol 2009; 8: 1128-39.

11. Dalgleish T. The emotional brain. Nat Rev Neurosci 2004; 5: 583-9.

12. Scott S, Caird FI, Williams BO. Evidence for an apparent sensory speech disorder in Parkinson's disease. J Neurol Neurosurg Psychiatry 1984; 47: 840-3.

13. Herrera E, Cuetos F, Rodríguez-Ferreiro J. Emotion recognition impairment in Parkinson's disease patients without dementia. J Neurol Sci 2011; 310: 237-40.

14. Beatty W, Goodkin D, Weir W, Staton R, Beatty PA. Affective judgments patients with Parkinson's disease or chronic progressive multiple sclerosis. Bull Psychol Soc 1989; 27: 361-4.

15. Jacobs DH, Shuren J, Bowers D, Heilman KM. Emotional facial imagery, perception, and expression in Parkinson's disease. Neurology 1995; 45: 1696-702.
16. Assogna F, Pontieri FE, Cravello L, Peppe A, Pierantozzi M, Stefani A, et al. Intensity-dependent facial emotion recognition and cognitive functions in Parkinson's disease. J Int Neuropsychol Soc 2010; 16: 867-76.

17. Suzuki A, Hoshino T, Shigemasu K, Kawamura M. Disgustspecific impairment of facial expression recognition in Parkinson's disease. Brain 2006; 129: 707-17.

18. Kan Y, Kawamura M, Hasegawa Y, Mochizuki S, Nakamura K. Recognition of emotion from facial, prosodic and written verbal stimuli in Parkinson's disease. Cortex 2002; 38: 623-30.

19. Lachenal-Chevallet K, Bediou B, Bouvard M, Thobois S, Broussolle E, Viguetto A, et al. Troubles de la reconnaissance des expressions faciales émotionnelles dans la maladie de Parkinson. Psychol Neuropsychiatr Vieil 2006; 4: 61-7.

20. Martins A, Muresan A, Justo M, Simao C. Basic and social emotion recognition in patients with Parkinson disease. J Neurol Sci Turkish 2008; 55: 247-57.

21. Clark US, Neargarder S, Cronin-Golomb A. Specific impairments in the recognition of emotional facial expressions in Parkinson's disease. Neuropsychologia 2008; 46: 2300-9.

22. Madeley P, Ellis A, Mihdham R. Facial expressions and Parkinson's disease. Behav Neurol 1995; 8: 115-9.

23. Adolphs R, Schul R, Tranel D. Intact recognition of facial emotion in Parkinson's disease. Neuropsychology 1998; 12 . 253-8.

24. Pell MD, Leonard CL. Facial expression decoding in early Parkinson's disease. Brain Res Cogn Brain Res 2005; 23: 327-40

25. Biseul I, Sauleau P, Haegelen C, Trebon P, Drapier D, Raoul S, et al. Fear recognition is impaired subthalamic nucleus stimulation in Parkinson's disease. Neuropsychologia 2005; 43: 1054-9.

26. Dujardin K, Blairy S, Defebvre L, Krystkowiak P, Hess U, Blond $\mathrm{S}$, et al. Subthalamic nucleus stimulation induces deficits in decoding emotional facial expressions in Parkinson's disease. J Neurol Neurosurg Psychiatry 2004; 75: 202-8.

27. Le Jeune F, Péron J, Biseul I, Fournier S, Sauleau P, Drapier S, et al. Subthalamic nucleus stimulation affects orbitofrontal cortex in facial emotion recognition: a PET study. Brain 2008; 131: 1599-608.

28. Péron J, Biseul I, Leray E, Vicente S, Le Jeune F, Drapier S, et al. Subthalamic nucleus stimulation affects fear and sadness recognition in Parkinson's disease. Neuropsychology 2010; 24: $1-8$.

29. Dujardin K, Blairy S, Defebvre L, Duhem S, Noël Y, Hess U, et al. Deficits in decoding emotional facial expressions in Parkinson's disease. Neuropsychologia 2004; 42: 239-50.

30. Breitenstein C, Daum I, Ackermann H. Emotional processing following cortical and subcortical brain damage: contribution of the fronto-striatal circuitry. Behav Neurol 1998; 11: 29-42.

31. Hoehn MM, Yahr MD. Parkinsonism: onset, progression and mortality. Neurology 1967; 17: 427-42.

32. Clark US, Neargarder S, Cronin-Golomb A. Visual exploration of emotional facial expressions in Parkinson's disease. Neuropsychologia 2010; 48: 1901-13.

33. Yip JTH, Lee TMC, Ho S, Tsang K, Li LSW. Emotion recognition in patients with idiopathic Parkinson's disease. Mov Disord 2003; 18: 1115-22.

34. Lawrence AD, Goerendt IK, Brooks DJ. Impaired recognition of facial expressions of anger in Parkinson's disease patients acutely withdrawn from dopamine replacement therapy. Neuropsychologia 2007; 45: 65-74.

35. Sprengelmeyer R, Young AW, Mahn K, Schroeder U, Woitalla D, Büttner T, et al. Facial expression recognition in people with medicated and unmedicated Parkinson's disease. Neuropsychologia 2003; 41: 1047-57.

36. Martínez-Corral M, Pagonagarraga J, Llebaria G, PascualSedano B, García-Sánchez C, Gironell A, et al. Facial emotion recognition impairment in patients with Parkinson's disease and isolated apathy. Parkinsons Dis 2010; 2010: 930627.

37. Calder AJ, Young AW, Keane J, Dean M. Configural information in facial expression perception. J Exp Psychol Hum Percept Perform 2000; 26: 527-51.

38. Assogna F, Pontieri FE, Caltagirone C, Spalletta G. The 
recognition of facial emotion expressions in Parkinson's disease. Eur Neuropsychopharmacol 2008; 18: 835-48

39. Cohen H, Gagné M, Hess U, Pourcher E. Emotion and object processing in Parkinson's disease. Brain Cogn 2010; 72: 457-63.

40. García-Rodríguez B, Casares-Guillén C, Molina J, Rubio G, Jurado-Barba R, Morales I, et al. Efectos diferenciales de la doble tarea en el procesamiento emocional en pacientes con enfermedad de Parkinson no medicados. Rev Neurol 2011; 53: 329-36.

41. McKinlay A, Grace RC, Dalrymple-Alford JC, Roger D. Characteristics of executive function impairment in Parkinson's disease patients without dementia. J Int Neuropsychol Soc 2010; 16: 268-77.

\section{Recognition of facial expression of emotions in Parkinson's disease: a theoretical review}

Introduction. Emotional facial expression is a basic guide during social interaction and, therefore, alterations in their expression or recognition are important limitations for communication.

Aim. To examine facial expression recognition abilities and their possible impairment in Parkinson's disease.

Development. First, we review the studies on this topic which have not found entirely similar results. Second, we analyze the factors that may explain these discrepancies and, in particular, as third objective, we consider the relationship between emotional recognition problems and cognitive impairment associated with the disease. Finally, we propose alternatives strategies for the development of studies that could clarify the state of these abilities in Parkinson's disease.

Conclusions. Most studies suggest deficits in facial expression recognition, especially in those with negative emotional content. However, it is possible that these alterations are related to those that also appear in the course of the disease in other perceptual and executive processes. To advance in this issue, we consider necessary to design emotional recognition studies implicating differentially the executive or visuospatial processes, and/or contrasting cognitive abilities with facial expressions and non emotional stimuli. The precision of the status of these abilities, as well as increase our knowledge of the functional consequences of the characteristic brain damage in the disease, may indicate if we should pay special attention in their rehabilitation inside the programs implemented.

Key words. Cognitive decline. Emotion recognition. Executive function. Facial expression. Parkinson's disease. Visuospatial abilities. 